\title{
TRANSTORNOS ALIMENTARES - QUADRO CLÍNICO
}

\author{
EATING DISORDERS-CLINICAL PICTURE
}

\author{
Nádia Juliana Beraldo Goulart Borges ${ }^{1}$, Juliana Maria Faccioli Sicchieri², Rosane Pilot Pessa Ribeiro ${ }^{3}$, \\ Júlio Sérgio Marchini ${ }^{4}$, José Ernesto Dos Santos ${ }^{4}$
}

\begin{abstract}
${ }^{1}$ Médica. Pós-Graduanda. Departamento de Clínica Médica. Faculdade de Medicina de Ribeirão Preto -USP. ${ }^{2}$ Nutricionista. Mestre. Escola de Enfermagem de Ribeirão Preto -USP. ${ }^{3}$ Docente. Departamento de Enfermagem Materno Infantil e Saúde Pública. Escola de Enfermagem de Ribeirão Preto-USP. ${ }^{4}$ Docente. Divisão de Nutrologia. Departamento de Clínica Médica. Faculdade de Medicina de Ribeirão Preto - USP

CoRRESPONDÊnCIA: Dra. Nádia Juliana Beraldo Goulart Borges.

Avenida do Café, 1149 apto 404 bloco C Vila Amélia- CEP 14050-230 Ribeirão Preto- SP

E-mail: nadiajborges@hotmail.com
\end{abstract}

Borges NJBG, Sicchieri JMF, Ribeiro RPP, Marchini JS, Dos Santos JE. Transtornos alimentares - Quadro clínico. Medicina (Ribeirão Preto) 2006;39 (3): 340-8.

RESUMO: A anorexia e bulimia nervosas são distúrbios da conduta alimentar caracterizados por abstenção voluntária de alimentos e pela ingestão compulsiva, seguida de métodos purgativos, respectivamente. Essas duas patologias estão intimamente relacionadas por apresentarem sintomas em comum: uma idéia prevalente envolvendo a preocupação excessiva com o peso, distorção da imagem corporal e um medo patológico de engordar. Geralmente, o perfil dos pacientes portadores de transtornos alimentares é: adolescentes do sexo feminino, raça branca, e alto nível sócio econômico cultural. Porém, o que se tem observado, é que esse grupo é cada vez mais heterogêneo, sendo realizado diagnóstico em adolescentes do sexo masculino, raça negra, pré adolescentes e pacientes com nível sócio econômico cultural baixo. Quanto à etiopatogenia, não há uma única etiologia responsável pela anorexia nervosa. Acredita-se no modelo multifatorial, com contribuição de fatores biológicos, genéticos, psicológicos, socioculturais e familiares. Neste trabalho apresentamos como se inicia o quadro de transtorno alimentar, critérios diagnósticos, diferenças clínicas entre anorexia e bulimia, complicações clínicas inerentes, diagnóstico diferencial, comorbidades psiquiátricas associadas e acompanhamento e evolução desses transtornos. Considerando a elevada prevalência dessas síndromes associada à alta morbidade, preconiza-se o melhor conhecimento de suas manifestações clínicas, bem como complicações associadas para que o diagnóstico possa ser realizado mais precocemente evitando assim que essas pacientes cheguem para o tratamento apenas quando seu estado já esteja grave.

Descritores: Transtornos da Alimentação. Anorexia Nervosa. Bulimia Nervosa.

\section{1- INTRODUÇÃO}

Os transtornos alimentares são doenças psiquiátricas caracterizadas por graves alterações do comportamento alimentar e que afetam, na sua maioria, adolescentes e adultos jovens do sexo feminino, podendo originar prejuízos biológicos, psicológicos e aumento da morbidade e mortalidade.
Os dois principais transtornos alimentares são a anorexia e bulimia nervosas.

A anorexia nervosa é caracterizada pela perda de peso à custa de dieta extremamente restrita, a busca desenfreada pela magreza, distorção da imagem corporal e alterações do ciclo menstrual.

A bulimia nervosa caracteriza-se por episódios repetidos de grande ingestão alimentar (episódios bu- 
límicos, do inglês "binge eating") e uma preocupação excessiva com o controle do peso corporal. O paciente chega a adotar medidas extremas, a fim de evitar o ganho de peso, devido à ingestão exagerada de alimentos. ${ }^{1}$

Essas duas doenças estão intimamente relacionadas por apresentarem alguns sintomas em comum: uma idéia prevalente envolvendo a preocupação excessiva com o peso, distorção da imagem corporal e um medo patológico de engordar.

Estudos epidemiológicos demonstram que há dificuldades relacionadas ao diagnóstico correto dos transtornos alimentares, como por exemplo, a recusa do paciente em procurar ajuda profissional ou por não admitir que está doente ou por achar que conseguirá se tratar sozinho. Com isso somente os casos mais graves procuram tratamento, o que pode implicar em incidência e prevalência subestimadas.

A incidência de novos casos de anorexia nervosa em mulheres jovens, de acordo com trabalhos nos Estados Unidos e Europa, pode variar de $1,43^{2}$ a $50^{3}$ por 100 mil pessoas anualmente. A incidência de bulimia nervosa é de 2 a $4 \%$ em países de primeiro mundo ${ }^{3}$.

Existe uma tendência ao aumento de incidência, em parte pela realização de um diagnóstico mais preciso por parte dos médicos ou da familiaridade com a doença por parte dos pais dos pacientes e por outro lado pela apologia atual do culto ao "corpo perfeito", inatingível pela maior parte da população.

A prevalência dos transtornos alimentares pode variar se considerarmos as síndromes parciais, isto é, pacientes que não apresentam a doença totalmente desenvolvida seja pela presença dos ciclos menstruais, seja pelo fato de a perda de peso não ser tão intensa quanto esperada.

Embora a anorexia se inicie na adolescência (geralmente entre 13 a 17 anos), casos com início na infância e após 40 anos têm sido observados. Na bulimia nervosa o início dos sintomas ocorre nos últimos anos da adolescência ou até os 40 anos, estando a idade média de início por volta dos 20 anos de idade. .,4 $^{1,4}$

Geralmente, o perfil dos pacientes portadores de transtornos alimentares é: adolescentes do sexo feminino, raça branca, e alto nível sócio econômico cultural. Porém, o que se tem observado, é que esse grupo é cada vez mais heterogêneo, sendo realizado diagnóstico em adolescentes do sexo masculino, raça negra, pré-adolescentes e pacientes com nível sócio econômico cultural baixo.
Quanto à etiopatogenia, não há uma etiologia única responsável pela anorexia nervosa. Acredita-se no modelo multifatorial, com contribuição de fatores biológicos, genéticos, psicológicos, socioculturais e familiares.

São ditos fatores de predisposição: sexo feminino, história familiar de transtorno alimentar, baixa auto-estima, perfeccionismo, dificuldade em expressar emoções.

Fatores precipitantes: dieta, separação e perda, alterações da dinâmica familiar, expectativas irreais, proximidade da menarca.

Fatores mantenedores: alterações endócrinas, distorção da imagem corporal, distorções cognitivas, práticas purgativas ${ }^{4}$.

\section{2- QUADRO CLÍNICO}

\section{1- Anorexia nervosa}

O início do quadro clínico ocorre freqüentemente a partir da elaboração de uma dieta, em que o paciente inicia a restrição de grupos alimentares, eliminando aqueles que julga mais calóricos. Essa restrição alimentar aumenta progressivamente, com diminuição do número de refeições, podendo evoluir drasticamente, até o jejum. O paciente tem como meta emagrecer, cada vez mais, desejando a todo custo ficar cada vez mais magro ${ }^{4}$.

Geralmente os pacientes relatam que o início do quadro se deu após um fator estressante como algum comentário sobre seu peso, ou o término de relacionamento, ou perda de ente querido. Paulatinamente o paciente passa a viver exclusivamente em função da dieta, do peso, da forma corporal, das atividades físicas, de tabela de calorias e do medo patológico de engordar. Concomitantemente esses pacientes apresentam traços de personalidade como preocupações e cautela em excesso, medo de mudanças, hipersensibilidade e gosto pela ordem.

Existem dois tipos de apresentação da anorexia nervosa: o restritivo e o purgativo. No primeiro, os pacientes utilizam comportamentos restritivos associados à dieta. Na anorexia tipo purgativa, acontecem episódios de compulsão alimentar, seguidos de métodos compensatórios, como vômitos auto induzidos e o uso de laxantes e diuréticos.

Entre os sintomas que podem ser referidos pelos pacientes estão: intolerância ao frio, fadiga, queda de cabelos, constipação, dor abdominal, anorexia, le- 
targia, pés e mãos frios, amenorréia, dificuldade de concentração, etc. Como em geral os pacientes não admitem estar doentes, eles tendem a não relatar espontaneamente suas queixas, ficando a cargo do médico de questioná-las.

Os achados clássicos no exame físico desses pacientes estão relacionados à desnutrição e à disfunção hipotalâmica e incluem pele seca, hipotermia, bradicardia, hipotensão, bradipnéia e edema de membros. A gravidade da desnutrição pode ser avaliada pelo índice de massa corpórea (IMC), que é obtido pela divisão do peso pelo quadrado da altura ( faixa de desnutrição inferior a $18 \mathrm{~kg} / \mathrm{m}^{2}$ para adultos) $)^{1,4,5}$.

\section{2- Bulimia nervosa}

Na bulimia nervosa, tipicamente o paciente começa a sentir uma vontade de comer incontrolável e, ao deparar-se com a geladeira, "devora" tudo. Sentese depois culpado e até mesmo mal estar físico em razão da quantidade ingerida de alimentos, ocorrendo-lhe a idéia de induzir o vômito para não engordar. Este comportamento lhe traz satisfação e alívio momentâneos. O paciente bulímico pensa em ter descoberto a forma ideal de manter o peso sem restringir os alimentos que considera proibidos. A progressão, todavia, é uma catástrofe. Após o vômito, surge a sensação de estar fazendo algo fora do normal. Sente-se ansioso, culpado e com piora na auto-estima, o que faz retomar a dieta às vezes de forma mais intensa por acreditar erroneamente que detém o controle sobre esse processo. Ao aumentar a restrição, facilita os episódios bulímicos, piora os vômitos, a ansiedade e a auto-estima virando um círculo vicioso ${ }^{6}$.

Os episódios bulímicos são definidos como "a ingestão, em um curto espaço de tempo, de uma quantidade de alimento muito superior ao que a maioria das pessoas conseguiria comer durante um período de tempo igual e sob circunstâncias similares"7. Além disso, deve haver um sentimento de perda de controle sobre a alimentação, ou seja, um sentimento de não poder parar de comer ou não controlar o quê e quanto se come ${ }^{7}$. A frequiência desses episódios é variável podendo ocorrer várias vezes em um único dia ou em uma semana.

Durante esses episódios, o paciente come sozinho e escondido, não dá atenção ao sabor e a textura do alimento, comendo sem nenhum critério na hora da compulsão. Porém o paciente é muito criterioso na escolha dos alimentos. Há também uma grande variabilidade nas calorias ingeridas durante o período da compulsão. Mitchell e Laine, em 1985, estudaram pacientes hospitalizadas com bulimia nervosa que descreveram seus episódios de "binge eating" (ingestão compulsiva seguida de vômitos). Esses pacientes relataram ingestões variadas de 1.436 a $25.755 \mathrm{Kcal}$, que eram muitas vezes semelhantes à dieta habitual. A compulsão alimentar não está somente relacionada ao número de calorias ingeridas, mas também aos sentimentos, perda de controle e ingestão de determinados tipos de alimentos. Alguns dos pacientes devoram o alimento até que a compulsão trouxesse dor física ou sono. A média dos episódios por semana foi treze (variando entre seis a trinta) ${ }^{8}$.

Wallin et al., em 1994, relataram que a seleção alimentar durante os episódios segue um padrão semelhante: pães, bolos, massas, sanduíches, chocolates, pizzas e doces. Segundo elas a seleção dos alimentos é resultado da "compulsão" ou do "desejo irresistível" por esse alimentos ${ }^{9}$. Segundo Hetherington e Rolls, 1991, o perfil da alimentação depende de uma série de fatores, incluindo a oportunidade de purgação, o tipo de alimento disponível e o humor ${ }^{10}$.

Mesmo não sendo a única prática usada para compensar o episódio bulímico ou para evitar ganho de peso excessivo, o vômito auto-induzido é o mais freqüentemente encontrado. Também é comum a prática intensa de exercícios físicos, podendo causar danos aos ligamentos e aos músculos. Também pode ocorrer o uso de laxantes, diuréticos, inibidores de apetite e de hormônios tireoidianos ${ }^{6}$.

Diferentemente do paciente anoréxico, o paciente bulímico não tem desejo de emagrecer cada vez mais. Em geral, seu peso está normal ou, em menor número de casos com sobrepeso. As irregularidades menstruais podem ocorrer, mas a amenorréia é característica da anorexia nervosa ${ }^{6}$.

É possível encontrar aumento da impulsividade, manifestada pela associação com outros quadros psiquiátricos, como cleptomania, tricotilomania e outros tipos de automutilação, abuso de drogas ilícitas e álcool, promiscuidade sexual e risco de suicídio ${ }^{11}$.

\section{3- CRITÉRIOS DIAGNÓSTICOS: DSM IV (anorexia e bulimia nervosas)}

O diagnóstico da anorexia e bulimia nervosas é feito segundo os critérios estabelecidos pelo DSM IV (Diagnostic and Statistical Manual of Mental Disorders; APA, 1994) ${ }^{7}$, conforme referido na Tabela I. 
Tabela I: Critérios diagnósticos dos transtornosa alimentares segundo DSM IV

\section{Anorexia nervosa}

1. Recusa em manter o peso corporal dentro ou acima do mínimo adequado a idade e a altura.

2. Medo intenso de ganhar peso ou tornar-se obeso, mesmo se abaixo do normal

4. Amenorréia em mulheres pós menarca (ausência de pelo menos três ciclos menstruais consecutivos)

\section{Subtipos:}

Restritivo: restrição dietética

Compulsivo/ Purgativo: ingestão excessiva/ vômitos, laxativos, diuréticos.
3. Distúrbio da imagem corporal

\section{Bulimia nervosa}

1. Episódios recorrentes de compulsão alimentar que pode ser caracterizada por:

a) comer, em um período de duas horas, grande quantidade de alimentos;

b) sentimento de perda de controle alimentar durante o episódio

2. Comportamento compensatório para prevenir o ganho de peso: vômitos auto-induzidos, abuso de laxantes, diuréticos, enemas ou outras drogas, jejum ou exercícios excessivos

3. A compulsão alimentar e comportamentos compensatórios ocorrem duas vezes/ semana, por, pelo menos, três meses

4. Preocupação excessiva com a forma corporal e o peso

5. O distúrbio não ocorre durante os episódios de anorexia nervosa

\section{Subtipos:}

Purgativo: vômitos ou abuso de laxativos, diuréticos e enemas

Não purgativo: jejum ou exercícios excessivos

\section{5- COMPLICAÇÕES CLÍNICAS DA ANO- REXIA E BULIMIA NERVOSAS}

A anorexia e bulimia nervosas são distúrbios da conduta alimentar caracterizados por uma obsessão pela perfeição do corpo e visão distorcida da imagem corporal.

Antes que se faça o diagnóstico de anorexia ou bulimia nervosas é importante excluir causas orgânicas que podem simular os quadros de transtornos alimentares como o descrito na Tabela II.
As complicações clínicas relacionadas à anorexia nervosa são decorrentes da própria desnutrição. Já na bulimia nervosa as complicações clínicas são mais frequientes, estando principalmente relacionado ao distúrbio hidroeletrolítico. No atendimento ambulatorial de pacientes portadores de transtornos alimentares do Hospital das Clínicas da Faculdade de Medi-

\section{Tabela II: Diagnóstico diferencial dos transtornos alimentares}

\section{Anorexia nervosa}

1. Doenças gastrointestinais e consumptivas (aids, câncer)

2. Síndrome da artéria mesentérica superior

3. Depressão e esquizofrenia

\section{Bulimia nervosa}

1. Anorexia nervosa do tipo compulsivo/ purgativo

2. Depressão com características atípicas

3. Distúrbios de personalidade

Adaptado: Distúrbios da conduta alimentar: anorexia e bulimia nervosa (Ribeirão Preto) $1988^{12}$ 
cina de Ribeirão Preto - USP, geralmente observamos uma prevalência aumentada de hipocalemia, sendo muitas vezes esse o motivo da internação de urgência. As complicações clínicas estão representadas na Tabela III.

\section{6- DIFERENÇAS CLÍNICAS ENTRE ANORE- XIA E BULIMIA}

A anorexia e bulimia nervosas apresentam diferenças clínicas significativas. Porém muitas vezes elas podem se confundir no subtipo purgativo da ano-

\section{Tabela III: Complicações clínicas da Anorexia e Bulimia}

\begin{tabular}{|c|c|c|}
\hline & Anorexia nervosa & Bulimia nervosa \\
\hline Pele e anexos & $\begin{array}{l}\text { Pele com aspecto amarelado pela } \\
\text { hipercarotenemia, pele seca, lânugo, } \\
\text { cabelos finos, unhas quebradiças, perda } \\
\text { de cabelo }\end{array}$ & $\begin{array}{l}\text { Calosidade no dorso da mão, pela } \\
\text { lesão da pele com os dentes ao } \\
\text { provocar o vômito (Sinal de Russel), } \\
\text { erosão do esmalte dentário, cáries. }\end{array}$ \\
\hline Sistema gastrointestinal & $\begin{array}{l}\text { Retardo do esvaziamento gástrico, } \\
\text { constipação, pancreatite, alteração de } \\
\text { enzimas hepáticas, diminuição do } \\
\text { peristaltismo intestinal }\end{array}$ & $\begin{array}{l}\text { Dor abdominal, gastrite, esofagite, } \\
\text { erosões gastroesofágicas, } \\
\text { sangramentos, constipação, prolapso } \\
\text { retal. Casos mais graves perfuração } \\
\text { esofágica e ruptura gástrica. }\end{array}$ \\
\hline Sistema cardiovascular & $\begin{array}{l}\text { Bradicardia,hipotensão, arritmias, } \\
\text { insuficiência cardíaca, miocardiopatia, } \\
\text { por ipepac, alterações do } \\
\text { eletrocardiograma, parada cardíaca }\end{array}$ & $\begin{array}{l}\text { Bradicardia,hipotensão, } \\
\text { arritmias,insuficiência cardíaca, } \\
\text { miocardiopatia por ipepac, alterações } \\
\text { do eletrocardiograma, parada cardíaca }\end{array}$ \\
\hline Sistema renal & $\begin{array}{l}\text { Edema, cálculo renal e elevação de } \\
\text { uréia devido à desidratação ou ao } \\
\text { catabolismo protéico }\end{array}$ & \\
\hline Sistema hematológico & Anemia, leucopenia, tromobocitopenia & \\
\hline Sistema reprodutivo & $\begin{array}{l}\text { Infertilidade, amenorréia, recém nascido } \\
\text { com baixo peso }\end{array}$ & Irregularidade menstrual \\
\hline Sistema metabólico & $\begin{array}{l}\text { Hipocalemia, hiponatremia, } \\
\text { hipoglicemia, desidratação }\end{array}$ & $\begin{array}{l}\text { Hipocalemia, } \\
\text { hipocloremia,hiponatremia, } \\
\text { hipoglicemia, alcalose } \\
\text { metabólica,desidratação }\end{array}$ \\
\hline Sistema endocrinológico & $\begin{array}{l}\text { Amenorréia, diminuição de } \\
\text { gonadotrofina, hormônio luteinizante e } \\
\text { estrogênio, aumento do hormônio de } \\
\text { crescimento e cortisol, diminuição do } \\
\text { T3 reverso, hipercolesterolemia }\end{array}$ & Irregularidade menstrual \\
\hline Outras alterações & $\begin{array}{l}\text { Hipotermia, intolerância ao frio, } \\
\text { convulsões, osteopenia/osteoporose, } \\
\text { alterações inespecíficas ao } \\
\text { eletroencefalograma }\end{array}$ & $\begin{array}{l}\text { Hipertrofia de glândulas parótidas } \\
\text { decorrentes dos vômitos, podendo ter } \\
\text { aumento da fração de amilase } \\
\text { produzida no local }\end{array}$ \\
\hline
\end{tabular}

Adaptado: Philippi, S.T. et al., $2004^{4}$ 
rexia nervosa. $\mathrm{O}$ aspecto em questão que auxilia no diagnóstico é que na anorexia do tipo bulímico o peso do paciente está abaixo do desejado enquanto que na bulimia ele é normal ou acima do desejável. A Tabela IV mostra diferenças entre os quadros de transtorno alimentar.

\section{7- AVALIAÇÃO LABORATORIAL DOS TRANSTORNOS ALIMENTARES}

Como o diagnóstico dos transtornos alimentares é clínico, não existem testes laboratoriais patognomônicos. Na verdade, as alterações encontradas são resultados dos hábitos utilizados para perder peso (p. ex. hipopotassemia devido vômitos excessivos).
É importante esclarecer à família que os exames laboratoriais solicitados são métodos para acompanhamento da doença e não para diagnóstico. Esta postura decorre do fato de que os pacientes não acreditam estar doentes e poderem se utilizar do fato dos exames estarem normais para justificar que não há nada de errado com eles 5 .

No Ambulatório de Transtornos Alimentares (GRATA-HCFMRP-USP) do Hospital das Clínicas da Faculdade de Medicina de Ribeirão Preto - USP, na primeira consulta solicita-se vários exames afim de diagnosticar possíveis alterações associadas aos transtornos alimentares. São eles:

- Hemograma: geralmente esses pacientes tem anemia hipocrômica microcítica leucopenia associada.

Tabela IV: Diferenças clínicas entre anorexia e bulimia

\section{Anorexia nervosa \\ Vômitos auto - induzidos no subtipo purgativo ou bulímico}

Abuso de diuréticos ou laxantes no subtipo purgativo ou bulímico

Perda de peso grave

Grave distorção da imagem corporal

Maior incidência aos 16 anos

Mais introvertidas

Negam fome

O comportamento alimentar é considerado normal pelo paciente e o desejo de controle de peso, justo e adequado

Sexualmente inativas

Amenorréia

Traços obsessivos de personalidade podem estar presentes

Comorbidade com doenças afetivas

Transtornos ansiosos

Impulsividade no subtipo purgativo

Adaptado: Philippi, S.T. et al., $2004^{4}$

\section{Bulimia nervosa}

Vômitos auto - induzidos no subtipo purgativo ou bulímico

Abuso de diuréticos ou laxantes no subtipo purgativo

Menor perda de peso, peso normal ou acima do normal

Quando existe é menos acentuada

Maior incidência aos 20 anos

Mais extrovertidas

Referem fome

O comportamento é motivo de vergonha, culpa, e há desejo de ocultá-lo

Mais ativas sexualmente

Menstruação variando de irregular à normalidade

Traços histriônicos e borderline podem estar presentes

Comorbidade com doenças afetivas e abuso de álcool e drogas

Impulsividade 
- Lipidograma: cerca de $40 \%$ dos pacientes com anorexia nervosa cursam com elevação do colesterol total ou podem apresentar diminuição do colesterol e triglicérides devido à própria desnutrição. Os possíveis mecanismos relacionados a hipercolesterolemia são: redução dos níveis de $\mathrm{T}_{3}$, diminuição da proteína carregadora do colesterol, perda do colesterol intra-hepático e diminuição da excreção fecal de ácidos biliares e colesterol ${ }^{5}$.

- Glicemia: geralmente esses pacientes cursam com hipoglicemia sendo que a exclusão do diagnóstico de diabetes melito é fundamental.

- Albumina e proteínas totais: esses pacientes podem apresentar hipoalbuminemia, sendo essa uma condição incomum devido à lenta e progressiva perda de peso.

- Eletrólitos sódio, potássio, cálcio iônico, magnésio)

- Zinco: muitas pacientes portadores de anorexia nervosa cursam com hipozinquemia que está relacionada à baixa ingestão alimentar.

- Estudo do ferro (ferro, ferritina, transferrina): para diagnóstico das anemias ferroprivas.

- Vitaminas (Vitaminas A, C, $\mathrm{B}_{12}$, ácido fólico e beta caroteno): diagnóstico de hipovitaminose relacionada à desnutrição.

- Testes tireoidianos: solicitamos TSH para triagem de hiper ou hipotireoidismo relacionada ao uso ou não de hormônios tireoidianos.

- Função renal: geralmente nesses pacientes os níveis de uréia e creatinina estão normais. Porém devido à desidratação ou ao catabolismo protéico esses valores podem se alterar.

- Função hepática: pacientes com anorexia nervosa podem cursar com esteatose hepática relacionada ao jejum.

- Eletrocardiograma: pacientes com transtornos alimentares podem cursar com bradicardia sinusal, arritmias sinusal ou ventriculares, principalmente devido ao prolongamento do intervalo QT. Caso se detecte essa alteração, deve-se monitorizar os níveis de eletrólitos desses pacientes, sobretudo se eles vomitam ou usam laxantes. A disfunção cardíaca mais séria é a taquiarritmia ventricular que pode resultar em morte súbita.

Esses pacientes podem cursar com outras alterações gonadais e do eixo hipotálamo-hipófise-adrenal além de alterações ósseas como a osteoporose. Neste sentido, os pacientes são avaliados não só do ponto de vista nutrológico, mas também do metabolismo intermediário.

\section{8- COMORBIDADES PSIQUIÁTRICAS AS- SOCIADAS AOS TRANSTORNOS ALI- MENTARES}

Na prática clínica observa-se que os transtornos alimentares se apresentam muitas vezes associados com outras doenças psiquiátricas. É descrito a associação com:

- Abuso e dependência de drogas e álcool (12-40\%)

- Síndromes depressivas (50-75\%)

- Transtornos ansiosos como fobia social e transtorno obsessivo compulsivo (15-35\%)

- Transtornos de personalidade (40-75\%) ${ }^{1}$.

\section{9- CURSO E EVOLUÇÃO DA ANOREXIA NERVOSA}

As taxas de recuperação da anorexia nervosa são variáveis. Estima-se, no entanto, que em torno de 30 a $40 \%$ dos pacientes tenham recuperação completa, não voltando a apresentar outros episódios da doença. Outros 30 a $40 \%$ têm uma evolução mediana, oscilando entre períodos de melhora e de recidiva da doença, podendo evoluir para bulimia nervosa ${ }^{13}$. O restante dos pacientes tem um curso grave, com complicações físicas e psicológicas mais sérias.

O índice de mortalidade pode chegar a $20 \%$, em razão das complicações decorrentes da própria doença e suicídio ${ }^{14}$.

Alguns fatores são preditivos de uma evolução desfavorável, como peso muito baixo no início do tratamento, aparecimento tardio da doença, demora para procurar ajuda médica, presença de práticas purgativas, relações familiares comprometidas e comorbidade psiquiátrica.

\section{0- CURSO E EVOLUÇÃO DA BULIMIA NER- VOSA}

De acordo com cada estudo, o critério de melhora é variável. Alguns autores ${ }^{15}$ consideram a bulimia recuperada, mesmo quando houver a ocorrência de um episódio bulímico ou vômito mensal, enquanto outros exigem o término completo da compulsão e das práticas purgativas.

Há boa recuperação em torno de $60 \%$ dos pacientes bulímicos, sendo $30 \%$ com evolução mediana e $10 \%$ com um curso ruim ${ }^{13}$.

Alguns fatores são considerados preditivos de mau prognóstico, tais como: grande freqüência de 
vômitos, aparecimento tardio da doença, maior severidade no quadro e relações interpessoais conturbadas. Porém, ainda cabem estudos prospectivos.

O tratamento tanto da anorexia quanto da bulimia nervosa deve ser multidisciplinar, com atendimento psiquiátrico, psicológico e nutricional, uma vez que vários fatores contribuem para o a aparecimento e a manutenção dos transtornos alimentares ${ }^{16}$.

Caso haja comorbidade psiquiátrica, é necessária a sua abordagem, em geral psicofarmacológica ${ }^{1,17}$.

No Grupo de Assistência em Transtornos Alimentares (GRATA) do Hospital das Clínicas da Faculdade de Medicina de Ribeirão Preto -USP, a abordagem inicial é feita a nível ambulatorial, com retornos frequientes. De acordo com a evolução clínica, esses retornos são reavaliados, agendado mais amiúde, postergado, ou se uma evolução clínica desfavo- rável, os pacientes são internados. Os critérios para a internação são: distúrbio hidroeletrolítico grave, ideações suicidas, quadros infecciosos, bradicardia, hipotermia, e peso menor que $75 \%$ do mínimo esperado. Além da adesão do paciente, é imprescindível, que a família esteja envolvida no tratamento. É oferecido assistência a família, com realização de grupos de apoio, para esclarecimento de dúvidas médicas, nutricionais e apoio psicológico, o que certamente influencia no bom prognóstico. Apesar da anorexia e bulimia nervosas, serem doenças com critérios clínicos bem definidos, os sistemas classificatórios não conseguem cobrir a variedade de alterações comportamentais alimentares, e, portanto há a necessidade de continuar a investigar e cuidar de pacientes portadores de transtornos alimentares clássicos, e dos que não apresentam os quadros tradicionais de tal transtorno.

Borges NJBG, Sicchieri JMF, Ribeiro RPP, Marchini JS, Dos Santos JE. Eating disorders - Clinical picture. Medicina (Ribeirão Preto) 2006; 39 (3): 340-8.

ABSTRACT: Anorexia and bulimia nervosa are eating disorders characterized by voluntary abstention and compulsive ingestion of food, followed by purgative methods, respectively. Both pathologies are related, once they present symptoms in common: a prevalent idea involving excessive preoccupation about weight, a distortion of body image, and a pathological fear of gaining weight. Generally, the profile of patients with eating disorders is: white female adolescents who have a high social, cultural and economic status. However, increasingly, such conditions have been involving other classes, including male Afro adolescents; pre-teens and patients who have lower social, cultural and economic status. As for the etiopathogeny of anorexia nervosa, there is not only one etiology behind it. A multifactor model is believed to exist, including family, social, cultural, psychological, economic, genetic, and biological factors. In this study we show how such conditions start, criteria for diagnoses, clinical differences between bulimia and anorexia, underling clinical complications, diagnoses differentials, related psychiatric comorbidities, progress, and follow up of such disorders. Considering the high prevalence and morbidity of such syndromes a better understanding of their manifestations and related complications is mandatory to reach early diagnoses. This way, patients will not seek treatment only when they already exhibit severe conditions.

Keywords: Eating Disorders. Anorexia Nervosa. Bulimia Nervosa.

\section{REFERÊNCIAS}

1 - Saito MI, Silva LEV. Adolescência: prevenção e risco. In: Cereser MG, Cordás TA. Transtornos alimentares: anorexia nervosa e bulimia. São Paulo: Atheneu; 2001. p. 269-76.

2 - Willi J, Giacometti G, Limacher B. Update on the epidemiology of anorexia nervosa in a defined region of Switzerland. Am J Psychiatry 1990; 147: 1514-7.
3 - Szmukler G, McCarice C, McCrone L, Hunter D. Anorexia nervosa: a psychiatry case register study from Aberdeen. Psychol Med 1986;16: 49-58.

4 - Philippi ST, Alvarenga M. Transtornos alimentares: uma visão nutricional. In: Cordás TA, Salzano FT, Rios SR. Os transtornos alimentares e a evolução no diagnóstico e no tratamento. Barueri: Manole; 2004. p. 39-62. 
5 - Vilar L, Castellar E. Endocrinologia clínica. In: Paula M, Teixeira L. Anorexia nervosa e outros transtornos alimentares. 2 th. ed. Rio de Janeiro: Medsi; 2001. p. 901-12.

6 - Fairburn CG, Cooper PJ. Eating disorders. In: Hawton K. Cognitive behaviour therapy for psychiatric problems. Oxford: Oxford University Press; 1989. p. 227-314.

7 - American Psychiatric Association. Diagnostic and statistical manual of mental disorders. $4^{\text {th }}$ ed. Washington, D. C.: APA; 1994

8 - Mitchell JE, Laine DC. Monitored binge eating behavior in patients with bulimia. Int J Eat Disord 1985; 4: 177-83.

9 - Wallin G, Van Der Ster, Noring C, Holmgren S. Binge eating versus nonpurged eating in bulimics: is there a carbohidrate craving after all?. Acta Psychiatr Scand 1994; 89: 376-81.

10 - Hetherington MM, Rolls BJ. Eating behavior in eating disorders: response to reloads. Physiol Behav 1991; 50: 101-8.

11 - McElroy SL, Kack PE, Phillips KA. Cleptomania, compulsive buying, and binge-eating disorder. J Clin Psychiatry 1995; 56 ( Suppl 4): 14-26.
12 - Ribeiro RPP, Santos PCM, Santos JE. Distúrbio da conduta alimentar: anorexia e bulimia nervosas. Medicina (Ribeirão Preto) 1998;31: 45-52.

13 - Herzog DB, Nussbaum KM, Marmor AK. Comorbidity and outcome in eating disorders. Psychiatr Clin North Am 1996; 19: $843-59$

14 - Theander S. Outcome and prognosis in anorexia nervosa and bulimia: some results of previous investigations compared with those of a Swedish long- term study. J Psychiatr Res 1985; 19: 493-508.

15 - Keel PK, Mitchell JE, Davis TL, Fieselman S, Crow SJ. Impact of definitions in the description and prediction of bulimia nervosa outcome. Int J Eat Disord 2000; 27: 377-86.

16 - Fisher M, Golden NH, Katzman DK, Kreipe RE, Rees J, Schebendach J, Sigman G, Ammerman S, Hoberman HM. Eating disorders in adolescents: a background paper. J Adolesc Health 1995; 16: 420-37.

17 - Braun DL, Sunday SR, Halmi KA. Psychiatric comorbity in patients with eating disorders. Psychol Med 1994; 24: 85967. 